\title{
Increased plasma concentrations of interleukin 35 in patients with coronary artery disease
}

\author{
Paulina Gorzelak-Pabiś, Maciej Chalubinski, Katarzyna Wojdan, Emilia Luczak, Iwona Duraj, \\ Monika Mozdzan, Marlena Broncel
}

Department of Internal Diseases and Clinical Pharmacology, Laboratory of Tissue Immunopharmacology, Medical University of Lodz, Lodz, Poland

Submitted: 28 January 2016

Accepted: 5 June 2016

Arch Med Sci 2017; 13, 4: 778-784

DOI: https://doi.org/10.5114/aoms.2016.63751

Copyright (c) 2016 Termedia \& Banach

\section{Abstract}

Introduction: Atherosclerosis leading to coronary artery disease (CAD) is a chronic inflammatory condition. Interleukin 35 (IL-35) released by regulatory T cells (Tregs) has been found to be associated with CAD in the Chinese population. However, nothing is known about the relation between IL-35 concentrations and cholesterol levels. The aim of the study was to assess the levels of IL-35 in CAD patients and healthy subjects from a Caucasian population, and to analyze the relationship between IL-35 and the levels of total cholesterol, low-density lipoprotein (LDL) and high-density lipoprotein (HDL) cholesterol, left ventricular ejection fraction (LVEF), sex and postmenopausal status. Material and methods: Thirty-one patients with CAD and 30 healthy controls were included in the study. Levels of plasma IL-35 were analyzed by ELISA. The LVEF was assessed by transthoracic echocardiographic examination. Plasma levels of cholesterol fractions and C-reactive protein (CRP) were assessed by immunoenzymatic methods.

Results: The CAD patients had higher levels of IL-35 as compared to healthy controls $(58.1 \pm 16.6 \mathrm{pg} / \mathrm{ml}$ vs. $5.35 \pm 3.35 \mathrm{pg} / \mathrm{ml} ; p<0.001)$. IL-35 levels negatively correlated with total and LDL cholesterol concentrations $(R=-0.31, p<0.01)$ and positively correlated with $\mathrm{HDL}$ cholesterol in men $(R=0.53, p<0.01)$. In women, IL-35 levels negatively correlated with LVEF $(R=-0.29, p<0.05)$ and positively with the duration of postmenopausal status $(R=0.55, p<0.01)$.

Conclusions: These results suggest a possible association between high levels of IL-35 and CAD.

Key words: interleukin-35, coronary artery disease, atherosclerosis, cholesterol, menopause, left ventricular ejection fraction.

\section{Introduction}

Atherosclerosis leading to the development of coronary artery disease (CAD) is a chronic inflammatory condition [1]. Many immune disorders are observed in atherosclerotic plaques, such as increased synthesis of the Th1-type cytokines interferon (IFN)- $\gamma$ and interleukin (IL)-12, which predominate over Th2 cell mediators [2, 3]. As an imbalance between T helper 1 (Th1)- and T helper 2 (Th2)-type cytokines does not fully account for the immune dysfunctions observed in atherogenesis, the immunomodulatory cytokines of other Th cell subsets may be potentially significant. According to Lin et al., patients with CAD demonstrate significantly lower plasma

\author{
Corresponding author: \\ Paulina Gorzelak-Pabiś MD \\ Department of Internal \\ Diseases and Clinical \\ Pharmacology Laboratory \\ of Tissue \\ Immunopharmacology \\ Medical University of Lodz \\ 1/5 Kniaziewicza St \\ 91-347 Lodz, Poland \\ Phone/fax: +48 426511059 \\ E-mail: kluzman.p@gmail.com
}


levels of IL-35 than patients with chest pain not associated with CAD [4].

Interleukin-35 is a cytokine first described in 2007 belonging to the IL-12 family, which comprises IL-12, IL-23 and IL-27 [5]. It consists of two subunits - the Epstein-Barr virus induced gene 3 (EBI3) protein and p35 - forming a heterodimer and is produced by activated CD4+CD25+foxp3+ regulatory $\mathrm{T}$ cells (Tregs) possessing immunosuppressive properties. Interleukin-35 functions as an anti-inflammatory agent inhibiting the proliferation of effector T cells $[4,6]$. Interleukin-35 has been shown to inhibit several Th1-mediated conditions in animal models of human chronic inflammatory diseases, such as experimental colitis $[5,7]$, collagen-induced autoimmune arthritis [8], demyelination of the central nervous system [9], as well as Th2-mediated allergic asthma [10]. Lin et al. propose that it may play a protective role in CAD [4]. Although EBI3 has been found in atherosclerotic plaques [11], the role of IL-35 in atherogenesis and CAD development has not been fully elucidated.

Interleukin-35 was shown to be associated with coronary artery disease in a Chinese population [4]. Therefore, the aim of this study was to analyze the plasma levels of IL-35 in CAD patients and healthy subjects in a Caucasian population. Additionally, the relationship between IL-35 levels and CAD risk factors, such as total cholesterol, low-density lipoprotein (LDL) and high-density lipoprotein (HDL) cholesterol levels, was assessed. Finally, the association of IL-35 with left ventricular ejection fraction (LVEF), sex and the duration of postmenopausal status was analyzed in CAD patients and healthy subjects.

\section{Material and methods}

\section{Patients}

The study included 61 adult subjects: a group of 31 coronary artery disease patients (12 females and 19 males, age $63.8 \pm 1.3$ ), and a control group of 30 healthy volunteers (15 females and 15 males, age $58.2 \pm 0.7$ ) (Table I A). The inclusion criterion was the presence of coronary artery disease, defined as at least $75 \%$ stenosis of one of the coronary arteries diagnosed by angiography of the coronary arteries by a cardiologist. The exclusion criteria were as follows: autoimmune diseases, allergy, immunodeficiency, neoplastic disease, immune response modifying drugs, such as glucocorticoids, cytostatics, monoclonal antibodies, tuberculosis, AIDS and HIV infection, viral hepatitis and fever. The criteria for exclusion from the control group were the presence of any symptoms of coronary artery disease in the history and ECG, and any other acute and chronic diseases. Plasma C-reactive protein (CRP) levels were assessed in all patients, and only those with a CRP below $10 \mathrm{mg} / \mathrm{l}$ were included in the study (Table | B). The informed consent was received from each patient included in the study. The clinical status of CAD patients is described in Table I A.

\section{Collecting and processing of blood samples}

Fasting blood samples were obtained by venous puncture, collected in sodium-heparin vacuum tubes and processed within $45 \mathrm{~min}$ by centrifugation for $20 \mathrm{~min}$ at $2200 \mathrm{~g}$. The supernatant was stored at $-80^{\circ} \mathrm{C}$. All samples were thawed only once.

\section{Interleukin-35 assessment in ELISA}

Plasma IL-35 levels were assessed by enzyme-linked immunosorbent assay (ELISA) using a ELISA ST-360 ELISA microplate reader $(450 \mathrm{~nm}$ and $630 \mathrm{~nm}$ ) according to the manufacturer's protocol (USCN Life Science Inc, E92008Hu). The range of IL-35 ELISA was $15.6-1000 \mathrm{pg} / \mathrm{ml}$.

Table I. Clinical characteristics of patients included in the study. A - Plasma concentrations and total cholesterol (TCH). All data are given as the mean $\pm \mathrm{SE}$, number of patients and percentage; statistical significance ${ }^{* *} p<0.01$. B - C-reactive protein (CRP) and left ventricular ejection fraction (LVEF) in patients with coronary artery disease (CAD) and HEALTHY controls (Spearman's $R$, statistical significance $\left.{ }^{*} p<0.05,{ }^{* *} p<0.01,{ }^{* * *} p<0.001\right)$

A

\begin{tabular}{|lcc|}
\hline Parameter & CAD $(n=31)$ & Healthy $(n=30)$ \\
\hline Age [years] & $63.8 \pm 1.3$ & $58.2 \pm 0.7^{* *}$ \\
\hline Gender (female/male) & $12 / 19$ & $15 / 15$ \\
\hline Hypertension, $n(\%)$ & $29(93)$ & $0(0)$ \\
\hline DM, $n(\%)$ & $8(25)$ & $0(0)$ \\
\hline Obesity, $n(\%)$ & $9(29)$ & $0(0)$ \\
\hline Nicotinism, $n(\%)$ & $6(19)$ & $0(0)$ \\
\hline
\end{tabular}

\section{B}

\begin{tabular}{|lcc|}
\hline Parameter & CAD $(n=31)$ & Healthy $(n=30)$ \\
\hline TCH $[\mathrm{mg} / \mathrm{dl}]$ & $166.1 \pm 9.3$ & $215.6 \pm 5.9^{\star * *}$ \\
\hline LDL $[\mathrm{mg} / \mathrm{dl}]$ & $96.4 \pm 7.5$ & $129.1 \pm 6.1^{\star * *}$ \\
\hline HDL $[\mathrm{mg} / \mathrm{dl}]$ & $50.1 \pm 3.0$ & $63.4 \pm 2.9^{\star * *}$ \\
\hline TG $[\mathrm{mg} / \mathrm{dl}]$ & $115.3 \pm 8.9$ & $115.6 \pm 9.5$ \\
\hline CRP $[\mathrm{mg} / \mathrm{l}]$ & $3.18 \pm 0.56$ & $2.1 \pm 0.4$ \\
\hline LVEF $(\%)$ & $50.0 \pm 2.3$ & $63.0 \pm 0.6^{* * *}$ \\
\hline
\end{tabular}

$C A D$ - coronary artery disease patients; Healthy - healthy individuals, control group. $N$ - number of patients in each group. $D M$ - diabetes mellitus, $L D L$ - LDL cholesterol, HDL - HDL cholesterol, TG - triglycerides. 


\section{Plasma C-reactive protein assessment}

Plasma CRP levels were assessed by immunoturbidimetry, amplified with latex molecules (Cobas c, Roche Diagnostics, USA) using a Roche/ Hitachi Cobas 6000 in the Wladyslaw Bieganski Hospital Laboratory, Lodz, Poland.

Plasma total cholesterol, LDL cholesterol, HDL cholesterol and triglycerides assessment

Plasma cholesterol and triglyceride levels were assessed enzymatically, while plasma HDL cholesterol concentration was assessed immunoenzymatically. A Cobas 6000 (Cobas Integra, Roche Diagnostics) instrument was used for all measurements in the Wladyslaw Bieganski Hospital Laboratory, Lodz, Poland.

\section{Left ventricular ejection fraction assessment}

Transthoracic echocardiographic examination was performed with MyLab 25 Gold, Esaote. Linear measurements were made according to the European Society of Echocardiography [12]. The LV volumes used to estimate ejection fraction (LVEF) were determined using the modified biplane Simpson's method. All echocardiographic measurements were performed by a cardiologist with subspecialty training in echocardiography. Reproducibility of echocardiographic measurements (interobserver and intraobserver bias) was assessed in the patients as a coefficient of variance from two sets of measurements made by two observers.

\section{Statistical analysis}

The results are presented as mean \pm SEM for variables with a normal distribution of values. The distribution of particular variables was verified by the Shapiro-Wilk W test, whereas the Levene test was performed to test homogeneity of variances. If the results demonstrated normal distribution and homogeneous variance, the significance of

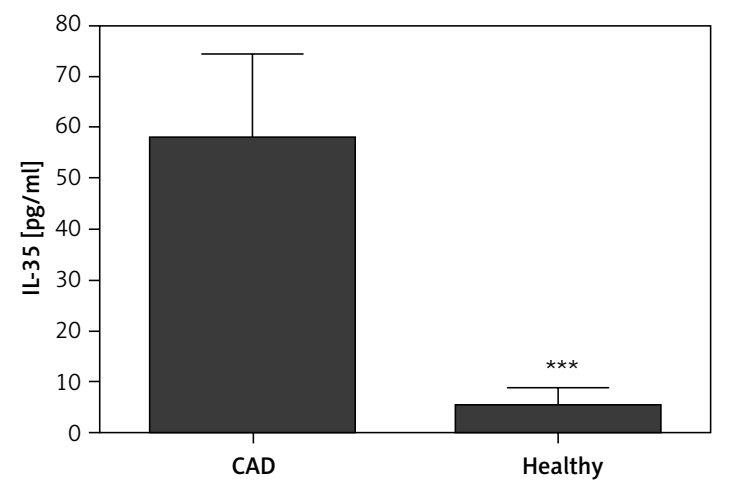

Figure 1. Plasma IL-35 concentrations in coronary artery disease patients (CAD) $(n=31)$ and healthy control group $(n=30)$; statistical significance ${ }^{* * *} p<0.001$ differences between 2 groups was estimated using the Student $t$-test for independent trials. However, if any of these criteria were not fulfilled, a Mann-Whitney $U$ test was used for analysis of the differences between the 2 groups. Spearman's correlation was used to calculate correlations between IL-35 levels and other measured parameters. In all tests, a value of $p<0.05$ was considered to be statistically significant.

\section{Results}

Plasma levels of IL-35 and CRP in coronary artery disease patients

Plasma concentrations of IL-35 were significantly higher in patients with CAD $(n=31)$ as compared to healthy donors $(n=30)(58.1 \pm 16.6$ $\mathrm{pg} / \mathrm{ml}$ vs. $5.35 \pm 3.35 \mathrm{pg} / \mathrm{ml} ; p<0.001$ ) (Figure 1). Moreover, out of 31 CAD patients, IL-35 was detectable in the sera of 21 (68\%) individuals, whereas 5 of $30(16 \%)$ healthy controls had detectible levels of IL-35. This indicates that the levels of IL-35 in most of the healthy subjects was very low. In order to exclude the presence of systemic acute or chronic inflammation, which may interfere with IL-35 levels and cause the difference in IL-35 concentrations between groups, patients with CRP below $10 \mathrm{mg} / \mathrm{l}$ were included in the study. There were no differences in CRP levels between CAD patients and healthy subjects (Table I B).

\section{Total cholesterol, LDL, HDL and triglyceride levels}

The levels of total cholesterol, LDL and HDL cholesterol and triglycerides, among the strongest risk factors of CAD, were assessed in the patients included in the study. Our results reveal that the total cholesterol and LDL concentrations in the sera were lower in the CAD patients $(n=$ $31)$ than the healthy subjects $(n=30)$ (166.1 $\pm 9.3 \mathrm{mg} / \mathrm{dl}$ vs. $215.6 \pm 5.9 \mathrm{mg} / \mathrm{dl} ; p<0.001$; and $96.4 \pm 7.5 \mathrm{mg} / \mathrm{dl}$ vs. $129.1 \pm 6.1 \mathrm{mg} / \mathrm{dl} ; p<0.001$, respectively). Lower levels of both total cholesterol $(T C H)$ and LDL in CAD patients occurred due to statin therapy. The CAD patients $(n=31)$ were also found to have lower levels of HDL $(50.1 \pm 3.0 \mathrm{mg} / \mathrm{dl}$ vs. $63.4 \pm 2.9 \mathrm{mg} / \mathrm{dl} ; p<0.001)$. No difference was observed in TG levels between the analyzed groups $(115.3 \pm 8.9 \mathrm{mg} / \mathrm{dl}$ vs. $115.6 \pm 9.5 \mathrm{mg} / \mathrm{dl}$; $p>0.05)$ (Table I B).

\section{Associations between plasma IL-35 \\ levels and total cholesterol, HDL and LDL cholesterol and triglyceride concentrations}

Next, we analyzed the relation between IL-35 plasma levels and CAD risk factors. Negative correlations were observed between IL-35 level and 
both the total cholesterol and LDL cholesterol concentrations in the whole group of patients included in the study $(R=-0.43, p<0.0001$, and $R=-0.31, p<0.01$, respectively; $n=61$ ). Conversely, a trend, however, non-significant statistically, was observed between IL-35 levels and $\mathrm{HDL}$ cholesterol concentrations in all patients $(R=0.23, p=0.06, n=61)$. However, while further analysis revealed that this correlation was negative in the group of women $(R=-0.34, p=0.07$, $n=61)$, this correlation was found to be strongly positive in men ( $R=0.53, p=0.001)$ (Table III). No association was found between II-35 levels and triglyceride concentrations $(n=61)$ (Table II).

\section{The association between LVEF and plasma IL-35 levels}

The LVEF was still significantly lower in CAD patients $(n=31)$ than in healthy controls $(n=30)$ $(50.0 \pm 2.3 \%$ vs. $63.0 \pm 0.6 \% ; p<0.001)$ (Table I B). Interestingly, a weak negative correlation was observed between IL-35 levels and LVEF in all subjects $(R=-0.29, p<0.05, n=61)$ (Table II), whereas further analysis revealed that while this correlation was stronger in the female subjects $(R=-0.48, p=0.01, n=27)$, no association between these parameters was observed in the male subjects $(R=0.2, p>0.05, n=34)$ (Table III).

\section{Associations between plasma IL-35 levels, age of the patients and duration of postmenopausal status in women}

A positive correlation was observed between IL-35 levels and age of patients included in the study $(R=0.26, p<0.05, n=61)$ (Table II). However, while this positive correlation was found to be much stronger in the women $(R=0.67, p=0.001$, $n=27$ ), no association between these two parameters was found in the men $(R=0.2, p>0.05$, $n=34$ ) (Table III). Further analysis revealed a positive correlation between the IL-35 levels and the duration of postmenopausal status in women $(R=0.55, p<0.01, n=27)$. Additionally, in the group
Table II. Association between plasma IL-35 levels and CAD risk factors, CRP, LVEF, age and duration of postmenopausal status in whole group of patients included in the study $(n=61)$

\begin{tabular}{|lcc|}
\hline IL-35 $[\mathrm{pg} / \mathrm{ml}]$ vs. & $R$ Spearman's & $P$-value \\
\hline TCH $[\mathrm{mg} / \mathrm{dl}]$ & -0.43 & $<0.001$ \\
\hline LDL $[\mathrm{mg} / \mathrm{dl}]$ & -0.31 & $<0.01$ \\
\hline HDL $[\mathrm{mg} / \mathrm{dl}]$ & 0.23 & 0.06 \\
\hline TG $[\mathrm{mg} / \mathrm{dl}]$ & -0.10 & $>0.05$ \\
\hline CRP $[\mathrm{mg} / \mathrm{l}]$ & -0.05 & $>0.05$ \\
\hline LVEF $(\%)$ & -0.29 & $<0.05$ \\
\hline Age $[$ years] & 0.26 & $<0.05$ \\
\hline
\end{tabular}

$T C H$ - total cholesterol, LDL - LDL cholesterol, HDL - HDL cholesterol, TG - triglycerides, CRP - C-reactive protein, LVEF - left ventricular ejection fraction (Spearman's $R$ - Spearman's correlation, $p$-statistical significance).

of postmenopausal women $(n=15)$ IL-35 plasma concentrations were found to be significantly higher as compared to premenopausal women $(n=12)$ $(59.4 \pm 18.7 \mathrm{pg} / \mathrm{ml}$ vs. $3.9 \pm 3.0 \mathrm{pg} / \mathrm{ml} ; p<0.01$ ) (Figure 2). Mean duration of post-menopausal status was $13.54 \pm 1.6$ years $(n=15)$.

\section{Discussion}

The present study demonstrates that patients with CAD have significantly higher plasma concentrations of IL-35 as compared to healthy individuals. This result contradicts those recently published by Lin et al., who report that IL-35 levels were significantly lower in stable angina patients as compared to individuals with chest pain syndrome not associated with CAD [4].

According to Lin et al., decreased IL-35 levels might be negatively associated with the inflammatory status of CAD patients [4]. Notably, in this study, subjects with stable angina not only had significantly higher CRP levels, reflecting the current inflammatory condition, than the control group, but they also displayed lower concentrations of IL-35. In our study, however, the levels of CRP were

Table III. Association between plasma IL-35 levels and CAD risk factors, CRP, LVEF, age and duration of postmenopausal status in women $(n=27)$ and men $(n=34)$

\begin{tabular}{|c|c|c|c|c|}
\hline \multirow[t]{2}{*}{ IL-35 [pg/ml] vs. } & \multicolumn{2}{|c|}{ Female $(n=27)$} & \multicolumn{2}{|c|}{ Male $(n=34)$} \\
\hline & $R$ Spearman's & $P$-value & $R$ Spearman's & $P$-value \\
\hline $\mathrm{HDL}[\mathrm{mg} / \mathrm{dl}]$ & -0.34 & 0.07 & 0.53 & $<0.01$ \\
\hline LVEF (\%) & -0.48 & 0.01 & 0.2 & $>0.05$ \\
\hline Age [years] & 0.67 & 0.001 & 0.14 & $>0.05$ \\
\hline $\begin{array}{l}\text { Post-menopause } \\
\text { [years of duration] }\end{array}$ & 0.55 & $<0.01$ & & \\
\hline
\end{tabular}

$T C H$ - total cholesterol, $L D L-L D L$ cholesterol, $H D L-H D L$ cholesterol, TG - triglycerides, CRP - C-reactive protein, LVEF - left ventricular ejection fraction (Spearman's $R$ - Spearman's correlation, $p$-statistical significance). 


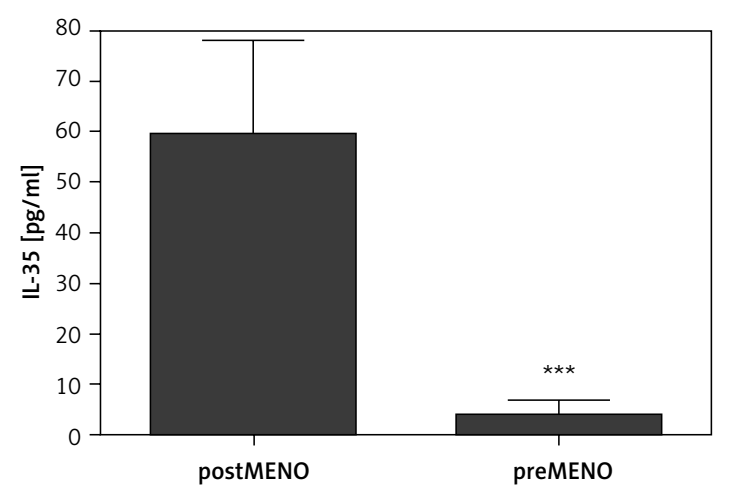

Figure 2. Plasma IL-35 concentrations in postmenopausal (postMENO, $n=12$ ) and premenopausal (preMENO, $n=15$ ) women; statistical significance ${ }^{* * *} p<0.01$

similar in CAD patients and healthy controls. Only patients with CRP below $10 \mathrm{mg} / \mathrm{ml}$ were included in the study in order to avoid the possibility of the presence of any inflammatory disease which could affect pro- and anti-inflammatory cytokine plasma concentrations. Therefore, the presence of systemic acute or chronic inflammation may be excluded as the cause of the difference in IL-35 levels between groups in our study.

Also, it may be the case that IL-35 is produced in higher amounts in CAD patients as a certain immune counteraction to the local release of inflammatory cytokines in order to decrease the activity of the immune processes ongoing within atherosclerotic plaques, thus serving as a physiological mechanism of diminishing the inflammation. This assumption may be supported by the finding that IL-35 expression may be induced by the inflammatory stimuli, such as tumor necrosis factor (TNF)- $\alpha$, interferon (IFN)- $\gamma$ and IL-1 $\beta$ [6], cytokines present in the atherosclerotic plaque. However, it is possible that this hypothetical mechanism is insufficient in CAD patients.

Additionally, one should be aware that IL-35 may be produced not only by Tregs, but also by the vascular endothelial cells and aortic smooth muscle cells [6]. Indeed, EBI3 subunit expression was found in smooth muscle cells of advanced atherosclerotic plaques [11], which is consistent with the higher levels of IL-35 found in the plasma of CAD patients in our study. What is more, stimulation of aortic smooth muscle cells with TNF- $\alpha$ and IFN- $\gamma$ was found to cause an increase of the expression of both EBI3 and p35 [11]. Therefore, it should be considered that the IL-35 measured in plasma of our patients may also be derived from the vascular wall tissues and may result from the activation of a reciprocal increase of IL-35 induced by proinflammatory TNF- $\alpha$ and IFN- $\gamma$.

As lipid disorders are among the most important risk factors of atherosclerosis and CAD development, the association between IL-35 and lipid levels was also assessed. A negative correlation was noted between IL-35 levels and both total cholesterol and LDL cholesterol concentrations in the whole group of patients, implying the existence of a certain relationship between these factors. However, it should be noted that these correlations, although statistically significant, are not strong. Interestingly, oxidized cholesterol has already been shown to also decrease the production of IL-10 [13], an anti-inflammatory cytokine. Moreover, treatment of hypercholesterolemic individuals with atorvastatin was evidenced to decrease LDL cholesterol concentrations and increase serum levels of IL-10 [14]. Notably, in our study, patients with CAD displayed lower levels of total cholesterol and LDL cholesterol as compared to the healthy group. Lower levels of both total cholesterol and LDL cholesterol in CAD patients could result from the statin therapy, which, according to the current guidelines, is a key and obligatory component of the pharmacological secondary prevention after myocardial infarction. Therefore, one cannot prejudge about the possible significance of the correlation between LDL cholesterol and IL-35.

There are almost no data in the literature on the effect of statins on IL-35 generation. Only atorvastatin has been shown to increase IL-35 levels in combination with methylprednisolone in the cerebrospinal fluid in multiple sclerosis patients as compared to methylprednisolone monotherapy [15]. As the majority of CAD patients included in the study were on statin therapy, one cannot exclude that it may have affected IL-35 levels. Further studies are needed.

High-density lipoprotein cholesterol levels were found to be significantly lower in CAD patients than in the healthy group. Additionally, a positive, however, non-significant trend of correlation was observed between IL-35 and HDL cholesterol levels $(p=0.06)$. Surprisingly, further analysis revealed that in the group of women, a positive, however, non-significant trend of correlation was negative $(p=0.07)$, whereas in men, it was found to be strongly positive $(p=0.001)$. Although there was no difference in IL-35 levels between women and men, HDL cholesterol concentrations were significantly higher in women (data not shown). Therefore, it could be speculated that HDL, contrary to LDL cholesterol, may positively regulate IL-35 synthesis in men.

Further analysis of the association between plasma IL-35 levels and the age of patients revealed certain differences related to gender. Firstly, the positive correlation between the IL-35 concentrations and age in women suggests that older male individuals tend to have higher IL-35 levels. We also observed that IL-35 plasma concentra- 
tions were higher in the group of postmenopausal women as compared to premenopausal women. Additionally, a positive correlation between IL-35 levels and the duration of the postmenopausal condition was observed. These results suggest that in women IL-35 levels may be partially regulated by sex hormones and influenced by the postmenopausal status, considered as an independent CAD risk factor [16]. This assumption may be supported by the results showing that postmenopausal women had higher levels of IL-10, TNF- $\alpha$ and IL-1 $\beta$ [17] and the synthesis of certain cytokines may be regulated by sex hormones. It has been shown that estrogens may modify the production of Th1, Th2 and Th17 cytokines by peripheral blood mononuclear cells [18]. Additionally, the release of the cytokines IL-2, IL-6, IL-10, IL-12, and IL-13 may be dependant on testosterone [19].

One may speculate on the explanation of the link between IL-35 levels and age and postmenopausal status in women, as aging itself is associated with immunosenescence. Numerous immune cell types in aged patients are characterized by uncontrolled release of cytokines and impairment of their activity as compared to young and healthy individuals. Elderly patients have a higher number of peripheral blood Tregs and an increased CD4/ CD8 ratio [20]. Furthermore, the Treg compartment expands with age, relative to the total T cells [2124]. Therefore, one cannot exclude that IL-35 levels in CAD patients may be partially associated with increased numbers of Tregs shown in other studies.

Finally, Lin et al. found a positive association between plasma IL-35 levels and LVEF in patients with CAD [5]. In contrast, our results reveal a negative correlation in women, and no relationship in men. In the light of these findings, IL-35 cannot be considered a biomarker of the prognosis of CAD, as postulated by Lin et al., at this early stage of investigation.

The number of patients included in our study may limit the final conclusions; however, it is close to the number in the study by Lin et al. in comparable groups. One possible cause of the discrepancy between our results and those obtained by Lin et al. could be the origin of the subjects: our study was conducted on Caucasians, while Lin et al. used Asian subjects for the study group. It cannot be excluded that the different genetic factors might be responsible for the different regulation of IL-35 in atherogenesis. The fact that IL-35 concentrations were detectible only in $16 \%$ of individuals from the healthy group and $68 \%$ of CAD patients also limits the final conclusions, especially when the associations between IL-35 levels and cholesterol levels are considered. Therefore, the issue of the possible involvement of IL-35 in atherogenesis requires further studies.
In conclusion, CAD patients have higher levels of IL-35 as compared to healthy controls. Interleukin-35 levels negatively correlate with both total cholesterol and LDL cholesterol concentrations, positively correlate with HDL in men and negatively correlate with LVEF in all patients. Additionally, IL-35 levels are higher in postmenopausal women and may be related to the duration of the postmenopausal status.

\section{Acknowledgments}

Ethical agreement: RNN/88/11/KE (May $17^{\text {th }}$ 2011). This study was supported by the Medical University of Lodz, Poland - grant no. 502-03/5165-01/502-54-013.

\section{Conflict of interest}

The authors declare no conflict of interest.

\section{References}

1. Hansson GK. Inflammatory mechanisms in atherosclerosis. J Thromb Haemost 2009; 7 Suppl 1: 328-31.

2. Zhong YX, Wang Q, Ji X, et al. CD4(+)LAP (+) and CD4 (+)CD25 (+)Foxp3 (+) regulatory T cells induced by nasal oxidized low-density lipoprotein suppress effector $T$ cells response and attenuate atherosclerosis in ApoE(-/-) mice. J Clin Immunol 2012; 32: 1104-17.

3. Hansson GK, Robertson AK, Soderberg-Naucler C. Inflammation and atherosclerosis. Annu Rev Pathol 2006; 1: 297-329.

4. Lin Y, Huang Y, Lu Z, et al. Decreased plasma IL-35 levels are related to the left ventricular ejection fraction in coronary artery diseases. PLoS One 2012; 7: e52490.

5. Collison LW, Workman CJ, Kuo TT, et al. The inhibitory cytokine IL-35 contributes to regulatory T-cell function. Nature 2007; 450: 566-9.

6. Li X, Mai J, Virtue A, et al. IL-35 is a novel responsive anti-inflammatory cytokine: a new system of categorizing anti-inflammatory cytokines. PLoS One 2012; 7: e33628.

7. Wirtz S, Billmeier U, McHedlidze T, Blumberg RS, Neurath MF. Interleukin-35 mediates mucosal immune responses that protect against T-cell-dependent colitis. Gastroenterology 2011; 141: 1875-86.

8. Niedbala W, Wei XQ, Cai B, et al. IL-35 is a novel cytokine with therapeutic effects against collagen-induced arthritis through the expansion of regulatory $T$ cells and suppression of Th17 cells. Eur J Immunol 2007; 37: 3021-9.

9. Zandian M, Mott KR, Allen SJ, Dumitrascu O, Kuo JZ, Ghiasi H. Use of cytokine immunotherapy to block CNS demyelination induced by a recombinant HSV-1 expressing IL-2. Gene Ther 2011; 18: 734-42.

10. Huang CH, Loo EX, Kuo IC, et al. Airway inflammation and IgE production induced by dust mite allergen-specific memory/effector Th2 cell line can be effectively attenuated by IL-35. J Immunol 2011; 187: 462-71.

11. Kempe S, Heinz P, Kokai E, Devergne O, Marx N, Wirth T. Epstein-Barr virus-induced gene- 3 is expressed in human atheroma plaques. Am J Pathol 2009; 175: 440-7.

12. Lang RM, Bierig M, Devereux RB, et al. Recommendations for chamber quantification. Eur J Echocardiogr 2006; 7: 79-108. 
13. Dushkin MI, Khoshchenko OM, Kudinova EN, Schwartz Y. Effects of hydroxysterols and atorvastatin on lipopolysaccharide-induced secretion of tumor necrosis factor and interleukin-10 by mouse macrophages. Bull Exp Biol Med 2006; 141: 233-5.

14. Hernandez C, Francisco G, Ciudin A, et al. Effect of atorvastatin on lipoprotein (a) and interleukin-10: a randomized placebo-controlled trial. Diabetes Metab 2011; 37: 124-30.

15. Li XL, Zhang ZC, Zhang B, et al. Atorvastatin calcium in combination with methylprednisolone for the treatment of multiple sclerosis relapse. Int Immunopharmacol 2014; 23: 546-9.

16. Gohlke-Barwolf C. Coronary artery disease: is menopause a risk factor? Basic Res Cardiol 2000; 95 Suppl 1: 177-83.

17. Vural P, Akgul C, Canbaz M. Effects of hormone replacement therapy on plasma pro-inflammatory and anti-inflammatory cytokines and some bone turnover markers in postmenopausal women. Pharmacol Res 2006; 54: 298-302.

18. Javadian A, Salehi E, Bidad K, Sahraian MA, Izad M. Effect of estrogen on Th1, Th2 and Th17 cytokines production by proteolipid protein and PHA activated peripheral blood mononuclear cells isolated from multiple sclerosis patients. Arch Med Res 2014; 45: 177-82.

19. Freeman BM, Mountain DJ, Brock TC, et al. Low testosterone elevates interleukin family cytokines in a rodent model: a possible mechanism for the potentiation of vascular disease in androgen-deficient males. J Surg Res 2014; 190: 319-27.

20. Johnstone J, Millar J, Lelic A, et al. Immunosenescence in the nursing home elderly. BMC Geriatr 2014; 14: 50.

21. Jagger A, Shimojima Y, Goronzy JJ, Weyand CM. Regulatory $T$ cells and the immune aging process: a minireview. Gerontology 2014; 60: 130-7.

22. Raynor J, Lages CS, Shehata H, Hildeman DA, Chougnet CA. Homeostasis and function of regulatory $T$ cells in aging. Curr Opin Immunol 2012; 24: 482-7.

23. Gregg R, Smith CM, Clark FJ, et al. The number of human peripheral blood CD4+ CD25high regulatory T cells increases with age. Clin Exp Immunol 2005; 140: 540-6.

24. Lages CS, Suffia I, Velilla PA, et al. Functional regulatory $T$ cells accumulate in aged hosts and promote chronic infectious disease reactivation. J Immunol 2008; 181: 1835-48. 